\title{
Calculation of intracranial pressure in human brain using Darcy's and Biot's models
}

\author{
J.-M. Drezet ${ }^{\mathrm{a}, \mathrm{b}} *$ and K. Shahim ${ }^{\mathrm{a}, \mathrm{b}}$ \\ ${ }^{a}$ Ecole Polytechnique Fédérale de Lausanne, Lausanne, Switzerland; ${ }^{b}$ LSMX, Station 12, CH-1015 Lausanne, Switzerland
}

Keywords: intracranial pressure; brain parenchyma; Darcy's permeability; Biot's model

\section{Introduction}

Cerebrospinal fluid (CSF) is a colourless liquid that fills the ventricular space and serves several functions like buoyancy, chemical stability and protection (Gideon et al. 1994). CSF is produced within the ventricles and partly by the ependyma cells. Part of it seeps towards the brain parenchyma where it is partially absorbed by the blood venous system. CSF flow disturbances lead to different brain diseases such as obstructive, communicating or normal pressure hydrocephalus (NPH). It is primordial to assess the influence of the CSF seepage through the ventricles towards the parenchyma on the ventricle deformation in a normal brain before studying the pathogenesis of NPH.

\section{Methods}

Similarly to Levine's (1999) work, Darcy's flow through the brain parenchyma is studied considering the brain as a fully saturated medium. The brain geometry is simplified and considered as a thick sphere with internal $\left(R_{\mathrm{i}}=2 \mathrm{~cm}\right)$ and external radius $\left(R_{\mathrm{e}}=8 \mathrm{~cm}\right)$. This assumption leads to the spherically symmetric problem where the analytical solution is sought under applied boundary conditions. Levine's (1999) analytical formulation is further extended to consider the CSF seepage towards the parenchyma through the ventricles. CSF is known to be produced by the choroid plexus at a maximum rate of $10^{-8} \mathrm{~m}^{3} / \mathrm{s}\left(\psi_{\max }\right)$; most of it goes to the aqueduct of Sylvius, whereas the rest flows through the brain parenchyma to the resorption region in the subarachnoid spaces (SAS; Welch 1963; Levine 1999; Linninger et al. 2009). CSF absorption by the blood vessels is modelled using Starling's law and assuming a uniform venous pressure, $p_{v}$ :

$$
\operatorname{div}\left(k_{\mathrm{D}} \vec{\nabla} p(r)\right)=k_{\mathrm{A}}\left(p(r)-p_{v}\right)
$$

where $k_{\mathrm{D}}$ and $k_{\mathrm{A}}$ are the brain tissue permeability and the absorption coefficients, respectively, and $p(r)$ is the interstitial CSF pressure (ICP). When introducing possible straining of the parenchyma, Biot's equation is obtained:

$$
\operatorname{div} \sigma+\vec{\nabla}\left(p(r)-p_{v}\right)=0
$$

where the stress $\sigma$ is defined using Hooke's elasticity law (Shahim et al. 2010). Over the pia layer $\left(r=R_{\mathrm{e}}\right)$, the displacement is zero due to the rigidity of the skull that constrains the parenchyma. All pressures are defined with respect to the venous pressure as suggested by Hakim et al. (1976) in one of their pioneering paper. ICP in the SAS, $p_{\text {pia, }}$, is reported to be very close to the venous pressure; in other words, the pia layer is assumed to be fully permeable thus imposing $p\left(R_{\mathrm{e}}\right)=p_{v}$. The boundary conditions consist in imposing the ventricle pressure and the CSF seepage flux $\psi$ on the ependyma membrane defined as $r=R_{\mathrm{i}}(\psi$ is positive and lower than $\psi_{\max }$ ):

$$
p\left(R_{\mathrm{i}}\right)-p_{v}=p_{\mathrm{vent}} \text { and }\left(\frac{\partial p}{\partial r}\right)_{R_{\mathrm{i}}}=\frac{-\psi}{4 \pi k_{\mathrm{D}} R_{\mathrm{i}}^{2}} .
$$

The solution $p(r)$ takes the following form:

$$
p(r)-p_{v}=\frac{A_{1}}{r} \sinh \left(\frac{r}{\sqrt{k}}\right)+\frac{A_{2}}{r} \cosh \left(\frac{r}{\sqrt{k}}\right),
$$

where $k=k_{\mathrm{D}} / k_{\mathrm{A}}$. Both coefficients $A_{1}$ and $A_{2}$ are defined by the two boundary conditions presented in Equation (3). By posing $p_{\text {pia }}$ equals $p_{v}$, one can calculate the transparenchymal ICP drop:

$$
\Delta p=\frac{\psi \sqrt{k} \times \tan h\left(\frac{R_{\mathrm{e}}-R_{\mathrm{i}}}{\sqrt{k}}\right)}{4 \pi R_{\mathrm{i}}^{2} k_{\text {darcy }}\left[1+\frac{\sqrt{k}}{R_{\mathrm{i}}} \tan h\left(\frac{R_{\mathrm{e}}-R_{\mathrm{i}}}{\sqrt{k}}\right)\right]} .
$$

The input parameters are brain permeability $k_{\mathrm{D}}=10^{-11} \mathrm{~m}^{4} / \mathrm{Ns}$, ventricular pressure $p_{\text {vent }}=1.1$ $\mathrm{mm} \mathrm{Hg}$, shear modulus $G=3 \mathrm{kPa}$ and Poisson's ratio $\nu=0.3$ (Shahim et al. 2010). Other parameters are determined hereafter.

\footnotetext{
*Corresponding author. Email: jean-marie.drezet@epfl.ch
} 


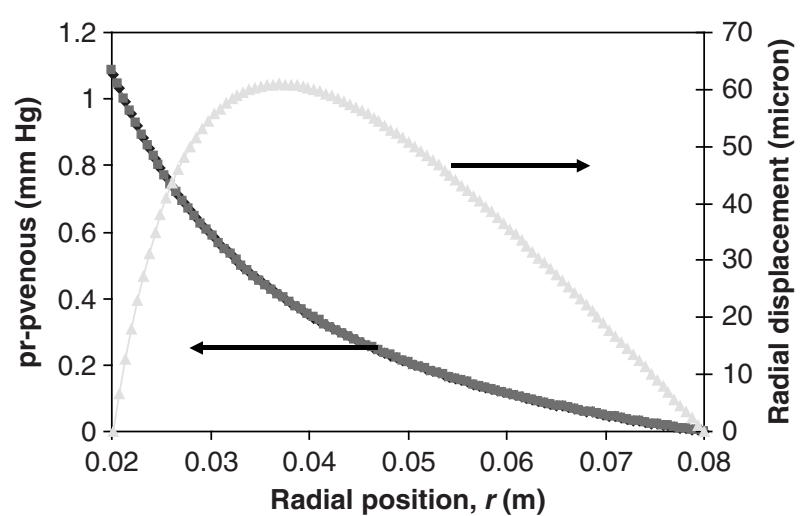

Figure 1. Calculated ICP distribution (left scale) using Darcy's and Biot's models and radial displacement (right scale).

\section{Results and discussion}

The absorption coefficient $k_{\mathrm{A}}$ is estimated by the fact that some of the CSF is flowing to the resorption sites of the pia layer. This leads to $\sqrt{k} \cong\left(R_{\mathrm{e}}-R_{\mathrm{i}}\right)$ thus yielding $k_{\mathrm{A}}=10^{-9} \mathrm{~m}^{2} / \mathrm{Ns}$. Using Biot's solution, the maximum seepage is $\psi=5 \times 10^{-10} \mathrm{~m}^{3} / \mathrm{s}=5 \% \psi_{\max }$ for a healthy brain exhibiting zero ventricle displacement at $r=R_{\mathrm{i}}$, i.e. no dilation, and a transparenchymal ICP drop of $1.1 \mathrm{~mm} \mathrm{Hg}$. The ICP radial distribution calculated with Darcy's model is presented in Figure 1 (left scale), and the radial displacement using Biot's model is shown on the right scale. The ICP distribution coming from Darcy's and Biot's models is identical. Indeed, the very slight radial straining that appears within the brain parenchyma with a maximum radial displacement of $60 \mu \mathrm{m}$ does not affect the pressure distribution. Most of the CSF entering the brain parenchyma is absorbed by the blood venous system but a small proportion can flow out the parenchyma towards the pia layer as the slope of pressure versus radial position is slightly negative at $r=R_{\mathrm{e}}$.

Owing to its elasticity, a healthy brain parenchyma can 'accept' CSF seepage of 5\% of the whole CSF ventricle production without deforming and still with keeping a very low transparenchymal pressure drop. If the CSF seepage increases by any reasons, ventricles deform and find another stable position at a radius higher than $2 \mathrm{~cm}$ thus leading to ventricle dilation. On the other hand, the brain parenchyma is put in triaxial tension at least within the periventricular region (Shahim et al., submitted) and thus exhibits a higher Darcy's permeability. As $k_{\mathrm{D}}$ increases, $\sqrt{k}$ becomes much larger than $\left(R_{\mathrm{e}}-R_{\mathrm{i}}\right)$ and Equation (5) simplifies to

$$
\Delta p=\frac{\psi\left(R_{\mathrm{e}}-R_{\mathrm{i}}\right)}{4 \pi R_{\mathrm{i}} R_{\mathrm{e}} k_{\mathrm{D}}}=\frac{\psi\left(R_{\mathrm{e}}-R_{\mathrm{i}}\right) \mathrm{e}^{-M \operatorname{tr} \varepsilon}}{4 \pi R_{\mathrm{i}} R_{\mathrm{e}} k_{\mathrm{D}}^{0}}
$$

with

$$
k_{\mathrm{D}}=k_{\mathrm{D}}^{0} \mathrm{e}^{M \operatorname{tr} \varepsilon}=10^{-11} \mathrm{e}^{4.3 \operatorname{tr} \varepsilon}\left(\mathrm{m}^{4} / \mathrm{Ns}\right)
$$

where $\operatorname{tr} \varepsilon$ is the trace of the strain tensor (Kaczmarek et al. 1997) that is calculated by solving Biot's equation. NPH is characterised by a large ventricle dilation and a pressure drop through the parenchyma close to normal, i.e. around $1 \mathrm{~mm} \mathrm{Hg}$. Equation (6) shows that if both $\psi$ and $k_{\mathrm{D}}$ increase in a similar manner, then $\Delta p$ can remain at a low value. In other words, in NPH condition, the increase of $\psi$ leads to an increase of $k_{\mathrm{D}}$ and both effects compensate to maintain a low transparenchymal pressure drop. When the CSF seepage reaches its maximum of $10^{-8} \mathrm{~m}^{3} / \mathrm{s}$, Biot's solution shows that the new value for $R_{\mathrm{i}}$ is $3.42 \mathrm{~cm}$, which is within the range of reported dilations. Note that $k_{\mathrm{D}}$ increases accordingly to $10^{-10} \mathrm{~m}^{4} / \mathrm{Ns}$.

\section{Conclusions}

A simple but realistic model of the CSF flow through the brain parenchyma is proposed by solving Darcy's and Biot's equations assuming spherical geometries for both ventricles and skull. It is shown that under healthy conditions, a small proportion, around $5 \%$, of CSF produced within the ventricles can seep towards the brain parenchyma without leading to ventricle dilation and be absorbed partially by the blood venous system. In that case, the transparenchymal pressure drop remains normal, i.e. around $1 \mathrm{~mm} \mathrm{Hg}$. If, for any reasons, the CSF seepage increases, then ventricle dilation appears and leads to the development of NPH as a new equilibrium radial position for the ventricle is reached. This conclusion is valid as long as the absorption coefficient is not affected by the deformation of the parenchyma. Further studies are needed to verify this assumption.

\section{Acknowledgement}

The financial support of the Swiss National Fund (SNF) No. 126753 is gratefully acknowledged.

\section{References}

Gideon P, Ståhlberg F, Thomsen C, Gjerris F, Sørensen PS, Henriksen O. 1994. Cerebrospinal fluid flow and production in patients with normal pressure hydrocephalus studied by MRI. Neurol. 36(3):210-215.

Hakim S, Venegas JG, Burton JD. 1976. The physics of the cranial cavity, hydrocephalus and normal pressure hydrocephalus: mechanical interpretation and mathematical model. Surg Neurol. 5(3):187-210.

Kaczmarek M, Subramaniam R, Neff S. 1997. The hydromechanics of hydrocephalus: Steady-state solutions for cylindrical geometry. Bull Math Biol. 59(2):295-323.

Levine D. 1999. The pathogenesis of normal pressure hydrocephalus: a theoretical analysis. Bull Math Biol. 61(5):875-916. 
Linninger AA, Sweetman B, Penn R. 2009. Normal and hydrocephalic brain dynamics: the role of reduced cerebrospinal fluid reabsorption in ventricular enlargement. Ann Biomed Eng. 37(7):1434-1447.

Shahim K, Drezet J-M, Molinari J-F, Sinkus R, Momjian S. 2010. Finite element analysis of normal pressure hydrocephalus: influence of CSF content and anisotropy in permeability. Appl Bionics Biomech. 7(3):187-197.

Shahim K, Drezet BM, Momjian S. Appl Bionics Biomech, submitted.

Welch K. 1963. Secretion of cerebrospinal fluid by choroid plexus of the rabbit. Am J Physiol. 205(3):617-624. 